\title{
Intelligent CAD System for Automatic Detection of Mitotic Cells from Breast Cancer Histology Slide Images Based on Teaching-Learning-Based Optimization
}

\author{
Ramin Nateghi, Habibollah Danyali, Mohammad Sadegh Helfroush, and Ashkan Tashk \\ Department of Electrical and Electronics Engineering, Shiraz University of Technology, Shiraz, Iran \\ Correspondence should be addressed to Ramin Nateghi; r.nateghi@sutech.ac.ir
}

Received 19 April 2014; Accepted 14 July 2014; Published 24 August 2014

Academic Editor: Giancarlo Mauri

Copyright (c) 2014 Ramin Nateghi et al. This is an open access article distributed under the Creative Commons Attribution License, which permits unrestricted use, distribution, and reproduction in any medium, provided the original work is properly cited.

\begin{abstract}
This paper introduces a computer-assisted diagnosis (CAD) system for automatic mitosis detection from breast cancer histopathology slide images. In this system, a new approach for reducing the number of false positives is proposed based on Teaching-Learning-Based optimization (TLBO). The proposed CAD system is implemented on the histopathology slide images acquired by Aperio XT scanner (scanner A). In TLBO algorithm, the number of false positives (falsely detected nonmitosis candidates as mitosis ones) is defined as a cost function and, by minimizing it, many of nonmitosis candidates will be removed. Then some color and texture (textural) features such as those derived from cooccurrence and run-length matrices are extracted from the remaining candidates and finally mitotic cells are classified using a specific support vector machine (SVM) classifier. The simulation results have proven the claims about the high performance and efficiency of the proposed CAD system.
\end{abstract}

\section{Introduction}

Nowadays, one of the most prevalent types of cancers which mostly lead to death is breast cancer [1]. Due to the World Health Organization (WHO) standardizations, there is a system known as Nottingham which is used for breast cancer grading. According to this system, three morphological features known as nuclear polymorphism, tubular formations, and number of mitosis cells are used for grading breast cancer [2]. The diagnosis of breast cancer grade is done by pathologists using histopathology slides. In recent years various computer-assisted diagnosis (CAD) systems on breast cancer diagnosis have been proposed. Such systems provide a great assistance for grading breast cancer samples faster and more accurately. In some of the researches, automatic breast cancer grading systems based on the three breast cancer grading features have been presented [3-5]. However, most of the researches on breast cancer histopathology images usually consider only one of the following: nuclear polymorphism $[4,6-8]$, tubule formations $[9,10]$, or mitosis cells counting [11-15]. The amount of dividing cells known as mitosis is essential feature for breast cancer grading. Pathologists count the number of mitoses in 10 distinct microscopic high power fields (HPFs) and, based on the average number of counted mitosis, the related scoring is calculated due to [5]:

$$
M C= \begin{cases}1 & N<9 \\ 2 & 10<N<19 \\ 3 & N>19\end{cases}
$$

where $N$ is the average number of mitoses in 10 HPFs.

For counting the number of mitoses in breast cancer histopathology slide images, several automatic methods have been proposed [11-15]. Some of these methods have used multispectral histopathology images for detecting and counting mitotic cells $[16,17]$. Khan et al. [11] proposed a method for automatic counting of mitotic cells from histopathology slide images in which a statistical gamma-Gaussian mixture model (GGMM) has been employed for estimating the probability density function (pdf) of mitosis and nonmitosis cells. By estimating pdfs of mitosis and nonmitosis, the potential mitosis candidates are extracted. Then, by training SVM classifier with the use of the statistical features extracted by 
Gabor filtering from all candidates, the mitoses are detected [11].

Sommer et al. [12] proposed another method in which the mitoses have been detected due to two level classifications: level one, in which random forest classification is employed to identify candidates, and level two, in which a SVM classification is applied to discriminate mitosis from nonmitosis objects. In another mitosis detection system, the original histopathology slide images are mapped from RGB to blueratio colour space [13]. In the blue-ratio colour space, the pixels belonging to the blue colour channel have higher grey scale values than the other two colour channels, that is, $R$ and $G$ colour channels. Due to Hematein staining effects, by using this mapping, the nuclei of cells, which have significant blue colour content, can be extracted using a simple thresholding approach. A Laplacian of Gaussian filtering having been applied to the blue-ratio image, a thresholding is applied to the filtered image. Consequently, initial candidates for mitosis objects are extracted. Then, by extracting different features such as cooccurrence and run-length matrices from each candidate and, by using specific classifiers, the mitoses are detected [13]. In some researches, for mitosis detection purpose, artificial neural networks (ANNs) [14] and exclusive independent component analysis (EICA) [15] have been employed. In some other more recently proposed papers such as [18-20], specific features with object-wise extraction considerations are proposed. This approach leads to better discrimination results between mitotic and nonmitotic objects. But, in general, some important challenges facing automatic mitosis detection from histopathology images exist that reduce accuracy of mitosis detection.

The organization of the rest of this paper is as follows. In Section 2, challenges facing the mitosis detection are described. Section 3 describes the proposed mitosis detection system which consists of candidate extraction, candidate selection, feature extraction and classification, and mitosis detection subsections. Experimental results to demonstrate effectiveness of our mitosis detection system are presented in Section 4 and finally the paper ends with a few concluding remarks in Section 5.

\section{Challenges Facing the Mitosis Detection}

In general, there are two challenges in front of automatic detection of mitosis cells from histopathology images. The first challenge is that the mitotic cells, existing in a histopathology image, normally have different color, shape, and texture. On the other hand, mitosis cells have a great deal of similarities with noncancerous cells and lymphocytes. The second challenge in front of appropriate mitoses detection is the high number of extracted candidates. In the other words, as the resolution of histopathology images is high and there are many other objects similar to mitoses, the number of extracted candidates is high.

In this paper, an automatic mitosis detection system is proposed that overcomes the stated challenges. To achieve this purpose, we extract different features based on color, texture, and shape of mitosis which overcome the first challenge. Also to overcome the second challenge, an approach

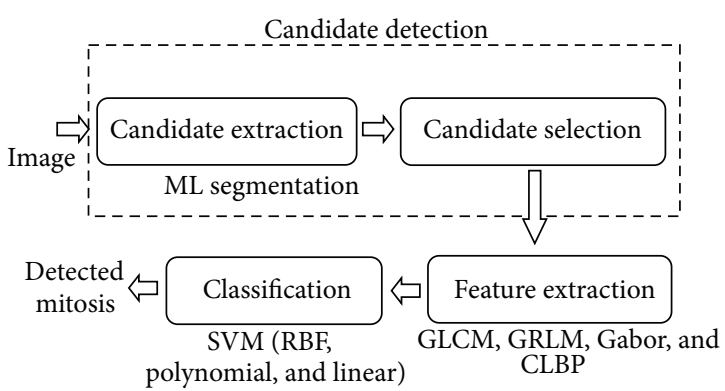

FIGURE 1: The proposed framework for mitosis detection.

for removing the large number of nonmitosis candidates is introduced. In the proposed method, the number of nonmitosis candidates is defined as a cost function for TLBO algorithm. By minimizing the defined cost function, a large number of nonmitosis candidates are omitted.

\section{Method}

Figure 1 shows the block diagram of the proposed system for automatic mitosis detection. In this system, the distribution parameter of mitosis and nonmitosis grey levels is estimated based on maximum likelihood (ML) algorithm. Using a ML algorithm and calculating a threshold that removes the background of histology image, the initial candidates are extracted that their counts are very high. In this section, an approach is proposed to reduce the high number of extracted nonmitosis candidates. After candidate selection procedure, color and texture features are extracted from remaining candidates and some features with significant discrimination abilities are applied to a support vector machine (SVM) classifier using different kernels for training. Finally, the trained classifier is used to classify the test candidates.

3.1. Candidate Extraction Using Maximum Likelihood ( $M L)$. In this subsection, the ML algorithm is used to estimate statistical probability density functions of mitosis and nonmitosis grey levels. In fact, by using training data, ML estimates the parameters of probability density functions related to mitosis and nonmitosis. As the pdf parameters are estimated by ML, proper thresholds could be calculated for candidate extraction. Assuming $x^{\prime}$ and $y^{\prime}$ as the grey level of mitosis and nonmitosis pixels, respectively, and also assuming mitosis and nonmitosis grey levels are independent of each other, the pdfs of mitosis and nonmitosis grey levels can be defined as

$$
\begin{aligned}
& p\left(X^{\prime} ; \theta_{X^{\prime}}\right)=p\left(x_{1}^{\prime}, x_{2}^{\prime}, \ldots x_{N}^{\prime} ; \theta_{x^{\prime}}\right)=\prod_{k=1}^{N} p\left(x_{k}^{\prime} ; \theta_{x^{\prime}}\right), \\
& p\left(Y^{\prime} ; \theta_{Y^{\prime}}\right)=p\left(y_{1}^{\prime}, y_{2}^{\prime}, \ldots y_{M}^{\prime} ; \theta_{y^{\prime}}\right)=\prod_{k=1}^{M} p\left(y_{k}^{\prime} ; \theta_{y^{\prime}}\right),
\end{aligned}
$$

where $X^{\prime}, Y^{\prime}$ are the grey levels of all mitosis and nonmitosis pixels.

The ML estimates the pdf parameters so that the mitosis and nonmitosis pdfs are maximized. According to Figure 2, 


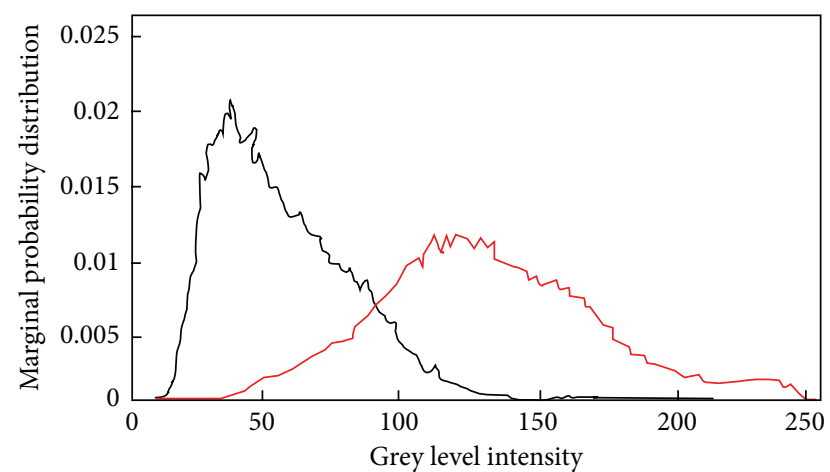

_ Marginal distribution (nonmitotic intensities)

_ Marginal distribution (mitotic intensities)

Figure 2: Pdfs of mitosis and nonmitosis grey levels.

the histogram of pixels belonging to mitosis and nonmitosis candidates is similar to Gaussian pdfs [11]. Gaussian probability density function has two parameters, mean $(\mu)$ and covariance $(\Sigma)$. These two parameters of Gaussian pdfs are estimated by ML as follows:

$$
\begin{array}{ll}
\mu_{x^{\prime}}=\frac{1}{N} \sum_{i=1}^{N} x_{i}^{\prime}, & \Sigma_{x^{\prime}}=\frac{1}{N} \sum_{i=1}^{N}\left(x_{i}^{\prime}-\mu_{x^{\prime}}\right)^{2}, \\
\mu_{Y^{\prime}}=\frac{1}{M} \sum_{i=1}^{M} y_{i}^{\prime}, & \Sigma_{Y^{\prime}}=\frac{1}{M} \sum_{i=1}^{M}\left(y_{i}^{\prime}-\mu_{y^{\prime}}\right)^{2},
\end{array}
$$

where $N$ is the number of mitotic pixels and $M$ is the number of nonmitotic ones.

As histopathology images are comprised of red, green, and blue components, therefore it is necessary that the mean and covariance parameters of mitosis and nonmitosis be estimated for separate $R, G$, and $B$ channels, separately. Thus, mean and covariance of mitosis and nonmitosis probability density functions have six $\mu_{X, R}, \mu_{X, G}, \mu_{X, B}, \Sigma_{X, R}, \Sigma_{X, G}$, and $\Sigma_{X, B}$ parameters that need to be estimated. As seen in Figure 2, by considering the intersection of mitosis and nonmitosis pdfs, it is possible to find thresholds for each colour component to extract the candidates. For example, threshold $T_{R}$ can be computed from (4), for the $R$ colour component,

$$
\begin{aligned}
& \exp \left(-\frac{\left(T_{R}-\mu_{x^{\prime}, R}\right)^{2}}{2 \Sigma_{x^{\prime}, R}}\right) \\
& =\exp \left(-\frac{\left(T_{R}-\mu_{y^{\prime}, R}\right)^{2}}{2 \Sigma_{y^{\prime}, R}}\right)
\end{aligned}
$$

$$
\begin{aligned}
& \Longrightarrow T_{R}=\left(\sigma_{x^{\prime}, R}^{2} \mu_{y^{\prime}, R}-\sigma_{y^{\prime}, R}^{2} \mu_{x^{\prime}, R}\right. \\
&+\left(\sigma_{x^{\prime}, R}^{2} \sigma_{y^{\prime}, R}^{2}\left(\mu_{x^{\prime}, R}-\mu_{y^{\prime}, R}\right)^{2}\right. \\
&\left.\left.+2\left(\sigma_{x^{\prime}, R}^{2}-\sigma_{y^{\prime}, R}^{2}\right) \ln \left(\frac{\sigma_{x^{\prime}, R}}{\sigma_{y^{\prime}, R}}\right)\right)^{1 / 2}\right) \\
& \times\left(\sigma_{x^{\prime}, R}^{2}-\sigma_{y^{\prime}, R}^{2}\right)^{2} .
\end{aligned}
$$

By obtaining the three thresholds, it is possible to extract initial candidates. According to this approach, three binary images including the mask of initial candidates are obtained due to

$$
C_{l}=\left\{\begin{array}{ll}
1, & \text { if } P_{l}(i, j)>T_{l} \\
0, & \text { otherwise }
\end{array} \quad l \triangleq R, G, B,\right.
$$

where $P_{x}(i, j)$ is the grey level of the pixel in coordinates $(i, j)$. For each threshold, three binary images named as $C_{R}, C_{G}$, and $C_{B}$ are achieved. Since for the next processing and also mitosis extraction, it is necessary that one of the extracted binary images comprising the mask of initial candidates be selected, thus, among $C_{R}, C_{G}$, and $C_{B}$, the best one that suffers from lower segmentation error is chosen. Segmentation error is defined as

$$
E_{l}=\sum_{i=1}^{n} \sum_{j=1}^{m} x \text { or }\left(C_{l}(i, j), G r(i, j)\right), \quad l \triangleq R, G, B,
$$

where $G r$ stands for the mitosis ground-truth binary mask. $n$ and $m$ are the height and width of $G r$, respectively. The candidates extracted by ML segmentation from a sample breast cancer HPF are shown in Figure 3(a). In the illustrated histopathology image, there are two mitoses, while the number of extracted candidates by ML segmentation is 356 (Figure 3(b)).

3.2. Candidate Selection. As seen in Figure 3, the number of candidates extracted by ML segmentation is often very high. In some other mitosis detection systems, this problem is worse. Although, in candidate detection stage of some previously proposed mitosis detection systems, statistical methods like gamma-Gaussian mixture model (GGMM) [11], pixel based classification [12], or colour mapping such as blueratio are employed, the number of extracted candidates is very high. This dilemma leads to the low accuracy of mitosis detection.

In the system introduced in this paper, a new approach toward reducing the number of nonmitosis candidates is proposed. Accordingly, the number of nonmitosis candidates will be considered as a cost function for an optimization algorithm. Minimizing of the cost function will lead to the reduction of a great number of nonmitosis objects as unwanted candidates. For this purpose, the mean of grey 


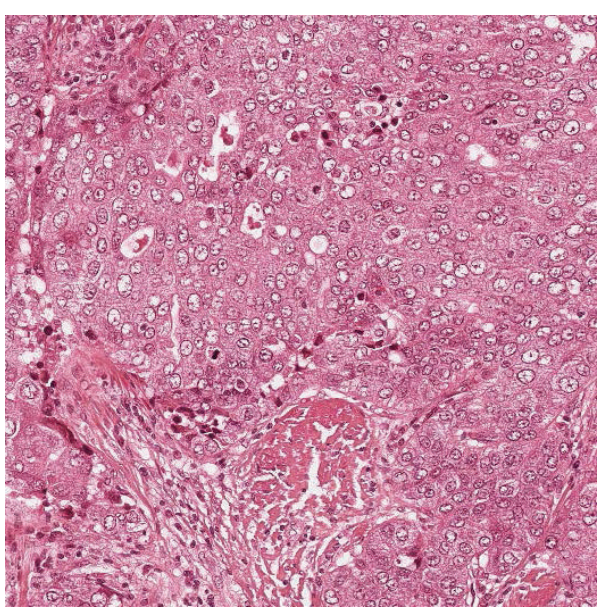

(a)

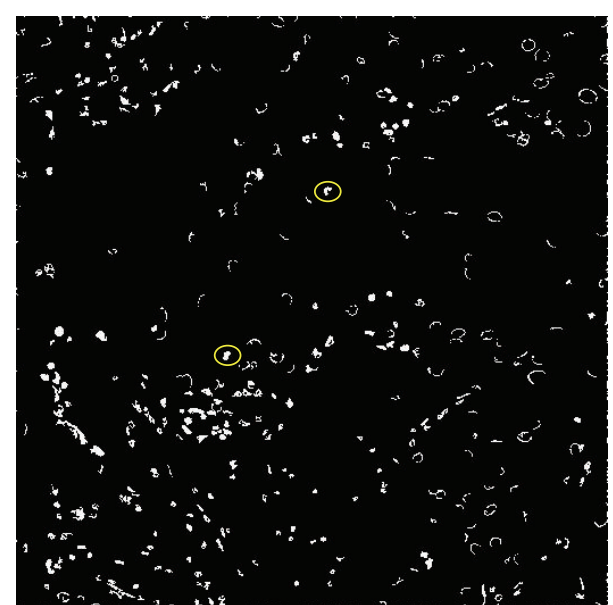

(b)

Figure 3: Detected candidates using ML segmentation: (a) a sample histopathology image that has six mitoses, (b) candidates extracted using ML algorithm.

levels is computed for each segmented candidate due to the following:

$$
M_{k}=\sum_{i=0}^{255} i \times p\left(x_{k}=i\right)
$$

where $M_{k}$ is the grey level mean and $p\left(x_{k}=i\right)$ relates to the histogram of $k$ th candidate. By computing $M_{k}$ for all candidates, the two vectors $\overline{\mathbf{M}}_{\mathbf{m}}$ (the mean of mitosis candidates) and $\overline{\mathbf{M}}_{\mathrm{nm}}$ (the mean of nonmitosis candidates) are obtained according to

$$
\begin{gathered}
\overline{\mathbf{M}}_{\mathbf{m}}=\left[M_{m 1}, M_{m 2}, \ldots M_{m L}\right], \\
\overline{\mathbf{M}}_{\mathbf{n m}}=\left[M_{n m 1}, M_{n m 2}, \ldots M_{n m P}\right],
\end{gathered}
$$

where $L$ and $P$ stand for the number of mitosis and nonmitosis candidates, respectively.

If it is assumed that there are $L+P$ candidates, then, by computing $T_{r}$ using (9), it is possible to eliminate a part of nonmitosis candidates. The number of nonmitosis candidates $\left(N_{r}\right)$, which can be removed, is computed according to (10)

$$
\begin{gathered}
T_{r}=\max \left(\overline{\mathbf{M}}_{\mathbf{m}}\right), \\
N_{r}=p_{r}\left(\overline{\mathbf{M}}_{\mathbf{n m}}>\operatorname{Tr}\right) .
\end{gathered}
$$

The main purpose is to provide a method to increase the number of eliminated nonmitoses candidates. For this reason, a weighting factor $h_{i}$ is assigned to the grey levels. The weighted mean is calculated as

$$
M_{k}^{\prime}\left(h_{i}\right)=\sum_{i=0}^{255} h_{i} \times i \times p\left(x_{k}=i\right), \quad h_{i} \sim\left[\begin{array}{ll}
0 & 1
\end{array}\right] .
$$

Due to this consideration, new vectors, $\overline{\mathbf{M}}_{\mathbf{m}}^{\prime}\left(h_{i}\right)$ and $\overline{\mathbf{M}}_{\mathbf{n m}}^{\prime}\left(h_{i}\right)$, will be obtained but the values of $h_{i}$ weights would not be known. To solve this problem, $h_{i}$ must be calculated so that $N_{r}$ has the maximum value. The maximum value of the $N_{r}$ is $P$. For computing the $h_{i}$ value, a cost function, $f$, which must be minimized, is defined as

$$
f\left(h_{i}\right)=P-N_{r}=P-p_{r}\left(\overline{\mathbf{M}}_{\mathbf{n m}}^{\prime}\left(h_{i}\right)>\mathbf{T}_{r}\right) .
$$

Solving this problem is not possible with deterministic optimization methods; therefore, to find an appropriate answer for $h_{i}$, teaching-learning-based optimization (TLBO) is employed. TLBO is a teaching-learning process-inspired algorithm proposed by Rao et al. [21] based on the effect of the teacher on the output of learners in a class. The algorithm describes two basic modes of learning: (I) via a teacher (known as the teacher phase) and (II) via interacting with the other learners (known as the learner phase). In this optimization algorithm, a group of learners is considered a population, and different subjects offered to the learners are considered design variables of the optimization problem. The best solution in the entire population is considered the teacher. The design variables are the parameters involved in the objective function of the given optimization problem, and the best solution is the best value of the objective function.

The procedures of TLBO algorithm are divided into two phases: the "teacher phase" and the "learner phase." In Algorithm 1, the steps of TLBO algorithm are shown consecutively [21]. Due to its simple concept and high efficiency, TLBO is a very attractive metaheuristic optimization technique and has been successfully applied to many real world problems [21]. Another important advantage of TLBO is that it does not have any control parameters.

By using training datasets and employing TLBO, the optimal values of $h_{i}$ weights and threshold $T_{r}$ can be obtained and it would be possible to reduce a large number of nonmitosis candidates in the mitosis candidate extraction phase.

If we assume that, in the test phase, there are a total number of $N_{t}$ candidates, according to (13), the candidates that their weighted means are greater than the threshold $T_{r}$ 


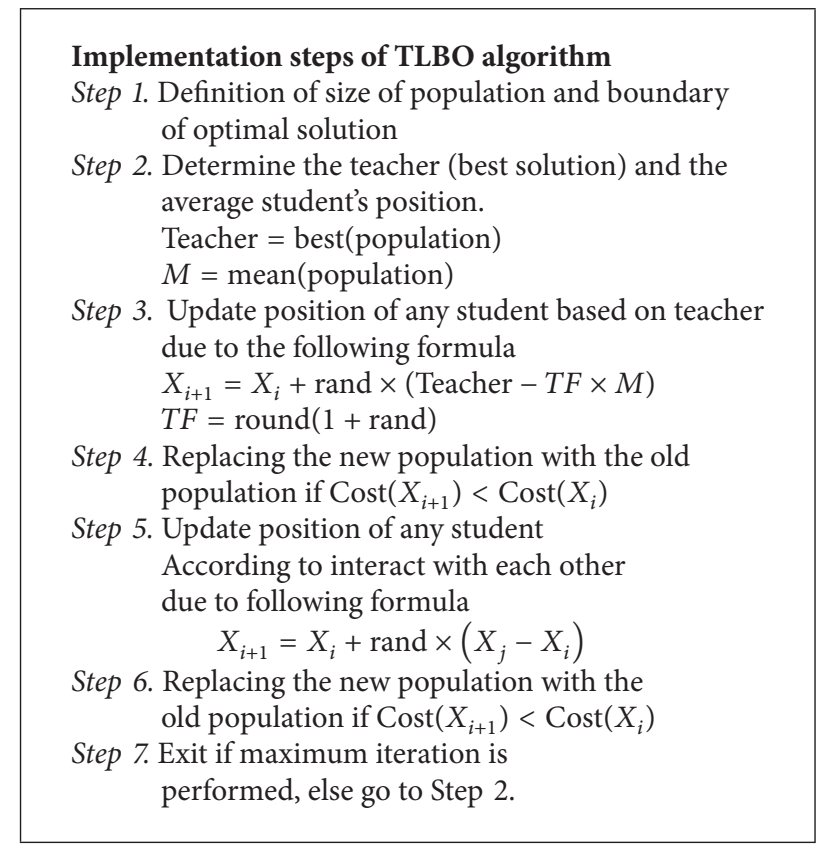

Algorithm 1: TLBO algorithm steps.

are omitted and the remaining candidates are passed to the next processing stages and final mitotic detection phase:

$$
\text { Candida }_{k}=\left\{\begin{array}{ll}
\text { removed, } & M_{k}^{\prime}>T_{r} \\
\text { remained, } & \text { otherwise }
\end{array} \quad k=1,2, \ldots, N_{t},\right.
$$

where $M_{k}^{\prime}$ is the weighted mean of $k$ th candidate which is achieved from (11).

3.3. Feature Extraction. For detecting and discriminating mitotic from nonmitotic candidates, some features based on colour, texture, and shape are extracted from the candidates remaining after candidate selection stage. As there are many similarities between mitotic cells and nonmitotic ones, the extracted features must be discriminant. The features that we extract are as follows: 14 haralick features derived from grey level cooccurrence matrices (GLCMs) [22], 11 features obtained from run-length matrices (CLRLMS) [23], 17 features achieved from complete local binary pattern (CLBP) with radius $R=2$ and the number of neighboring pixels $p=16$ [24], 4 statistical features comprising grey level mean, variance, and third and fourth moments, 32 mean and energy features obtained from two level decomposition of packet wavelet [25], and 32 energy features obtained from Gabor filtering based on 8 directions and 4 frequencies [26]. Since the histopathology images are RGB, the features are extracted from the three colour components; therefore the final length of feature vectors is 330 .

3.4. Classification and Mitosis Detection. According to the extracted feature vectors which are used for training a SVM classifier, the mitosis classification and detection are executed. During the SVM classification, the input feature data are mapped into a higher order space. By this mapping, which is usually done based on a kernel, the SVM is able to discriminate the feature vectors by optimal hyperplanes in higher order space. It is proved that, in a SVM for calculating optimal hyper planes, the following formula must be calculated:

$$
\begin{gathered}
\max _{a_{i} \geq 0}\left\{\sum_{i=1}^{n} a_{i}-\frac{1}{2} \sum_{i, j=1}^{n} y_{i} y_{j} a_{i} a_{j} K\left(x_{i} \cdot x_{j}\right)\right\}, \\
\sum_{i=1}^{n} a_{i} y_{i}=0
\end{gathered}
$$

where $a_{i}$ is Lagrangian coefficient and $x_{i}$ is related to the data of each class and $y_{i}$ are the output of the SVM classifier and $K\left(x_{i}, x_{j}\right)$ is the kernel function [27].

In general, the kernel functions may be linear, radial basis function (RBF), homogeneous and inhomogeneous polynomials, and also sigmoid kernels [24].

In this paper, three different types of kernels, that is, $\mathrm{RBF}$, polynomial, and linear, are used for mitosis detection. SVM algorithm is very sensitive to the entity of the chosen training and testing data; therefore, a $k$-fold cross-validation is applied to the randomly selected training and testing data and accordingly the SVM classifier is used many times. In this approach, data $F$ are divided into $K$ parts. Then, $K-1$ parts of the data $F$ are used as training dataset $\left(F_{\mathrm{tr}}\right)$ and the remaining part $\left(F_{\text {te }}\right)$ is used as testing dataset. This procedure is repeated $k$ times and, at each step, new $F_{\text {tr }}$ and $F_{\text {te }}$ dataset are generated and the classification becomes accomplished. The mean accuracy obtained through the $K$ repetition of SVM classifier is considered as the final mitosis detection accuracy. 


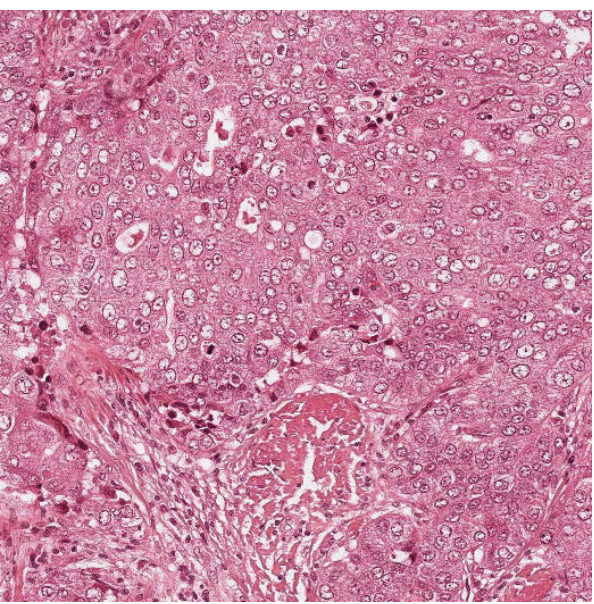

(a)

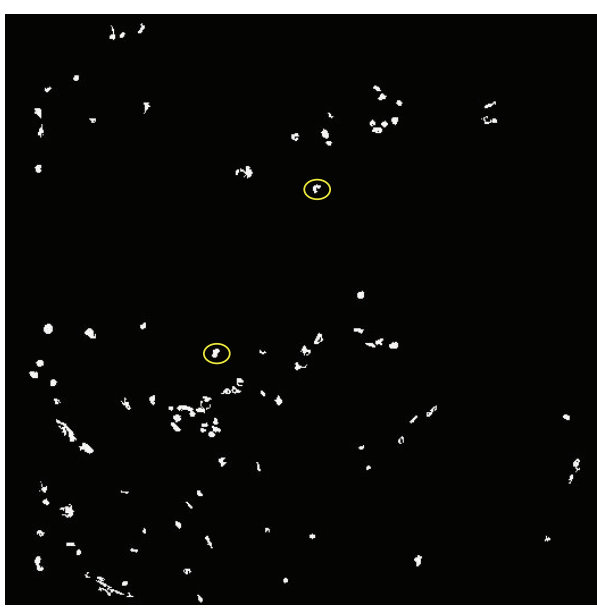

(c)

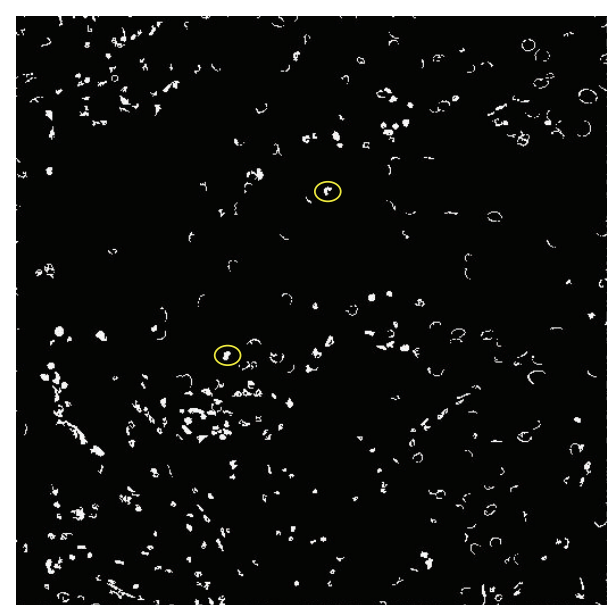

(b)

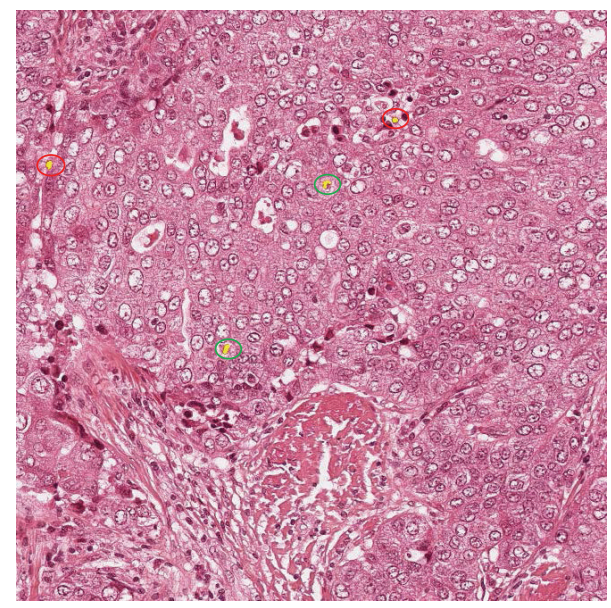

(d)

Figure 4: The proposed mitosis detection results: (a) sample breast cancer histopathology image, (b) detection candidates using ML algorithm, (c) selection of desired candidates and removing of most of the nonmitosis candidates, and (d) result of mitosis detection: green circles are true positive results; red circle shows false positive one.

\section{Result and Discussions}

The database employed for simulations is related to ICPR 2012 mitosis detection contest [28] that includes 35 breast cancer histopathology slide images which are acquired by Aperio XT scanners with 40x magnification. The resolution of histopathology images is $2084 \times 2084$ pixels. The total numbers of mitoses among 35 database images are 236. For training SVM classifier and achieving the results, we use $k$ fold where the parameter $k$ and the number of iteration are set to 5 and 100, respectively.

For evaluating the results, there are some specific accuracy measurement criteria known as precision, recall, and Fmeasure which are defined by (15) to (17). Precision criterion describes how many of the final classified candidates are real mitoses. This criterion is defined due to (15) in which TP (true positive) stands for the number of truly detected mitoses. FP stands for the false positive (those candidates related to the number of nonmitoses that are misclassified as mitosis).
The less the amount of FP is, the greater the precision will be. Recall or sensitivity criterion explains how much the algorithm is successful in detecting more numbers of mitosis correctly or, in fact, how much it is successful to avoid losing real mitosis candidates existing in the original image. This criterion is defined as (16) in which FN (false negative) stands for the number of the lost mitosis objects. The less the numbers of FN are, the higher the sensitivity criterion will be. Moreover, F-measure is the harmonic mean of the two previous criteria which measures the reconstruction degree between precision and recall criteria:

$$
\begin{gathered}
\text { Precision }=\frac{T_{P}}{T_{P}+F_{P}}, \\
\text { Recall }=\frac{T_{P}}{T_{P}+F_{N}}, \\
\text { F-score }=\frac{2 \times \text { Precision } \times \text { Recall }}{\text { Precision }+ \text { Recal }} .
\end{gathered}
$$




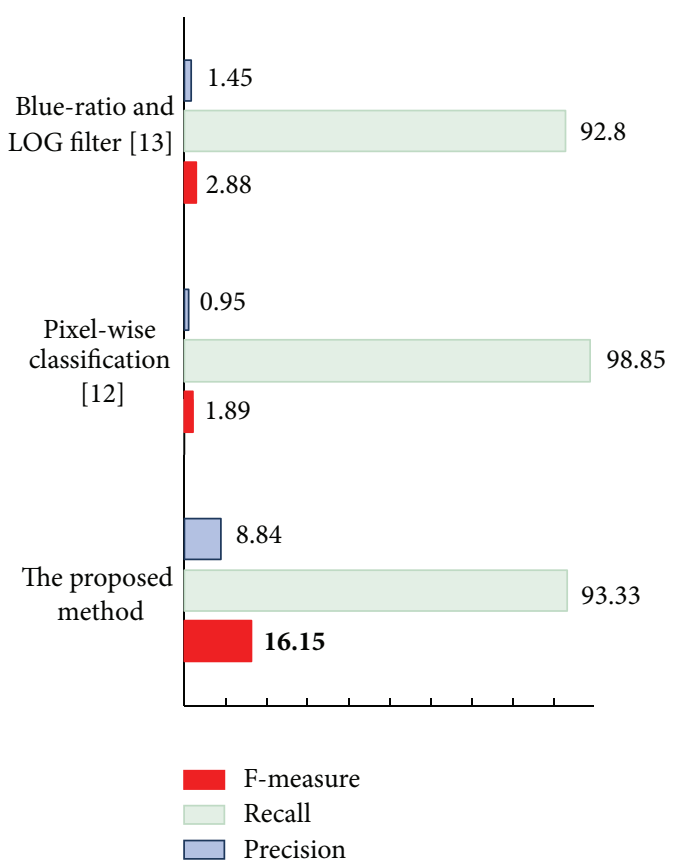

FIGURE 5: Candidate extraction efficiency compared to different algorithms.

4.1. Efficiency of the Proposed Mitosis Detection System. The results obtained by the proposed method are discussed and compared with the results of other algorithms from two aspects: number of extracted candidates and mitosis detection accuracy. Based on the F-measure criteria, the efficiency of the candidate extraction stage in the proposed method is compared with candidate extraction stage of other mitosis detection systems such as Laplacian of Gaussian filtering [13] and pixel-wise classification [12]. In candidate extraction stage of the proposed mitosis detection system, the training datasets are employed and the TLBO algorithm with initial population of $N=500$ under 1500 iterations is performed. The number of initial candidates achieved by ML estimation for testing datasets is about 6956 . By the means of the proposed method that removes most of the nonmitosis candidates, the number of remaining candidates after reduction is reduced to 950 which means that about 86 percent of nonmitosis candidates are removed. Figure 5 compares the efficiency of the candidate extraction stage of the proposed method with two other systems based on the precision, recall, and F-measure criteria. The proposed algorithm resulted in $8.84 \%$ precision, $93.33 \%$ recall, and $16.15 \% \mathrm{~F}$-measure. The proposed algorithm in eliminating the nonmitotic candidates is much better than existing algorithms.

Figure 6 shows mitosis detection implementation results of the proposed system based on different SVM kernels. Because of the high overlapping between the feature vectors belonging to mitosis and nonmitosis classes, the RBF kernel, which projects the data to a higher order space nonlinearly, has better classification results than the linear and polynomial kernels. Based on the precision, recall, and F-measure criteria, the efficiency of the proposed mitosis detection method is

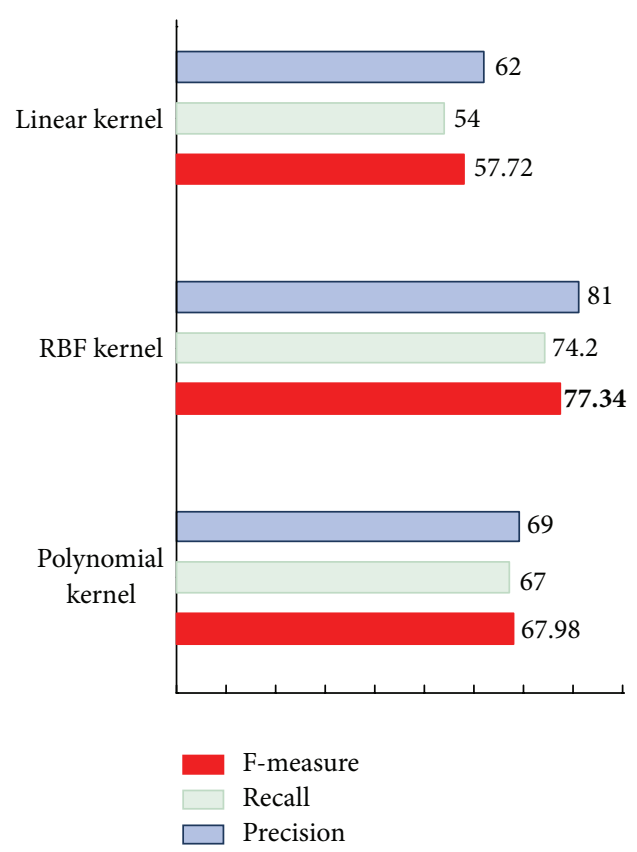

FIGURE 6: Result of the proposed mitosis detection system using different SVM kernel.

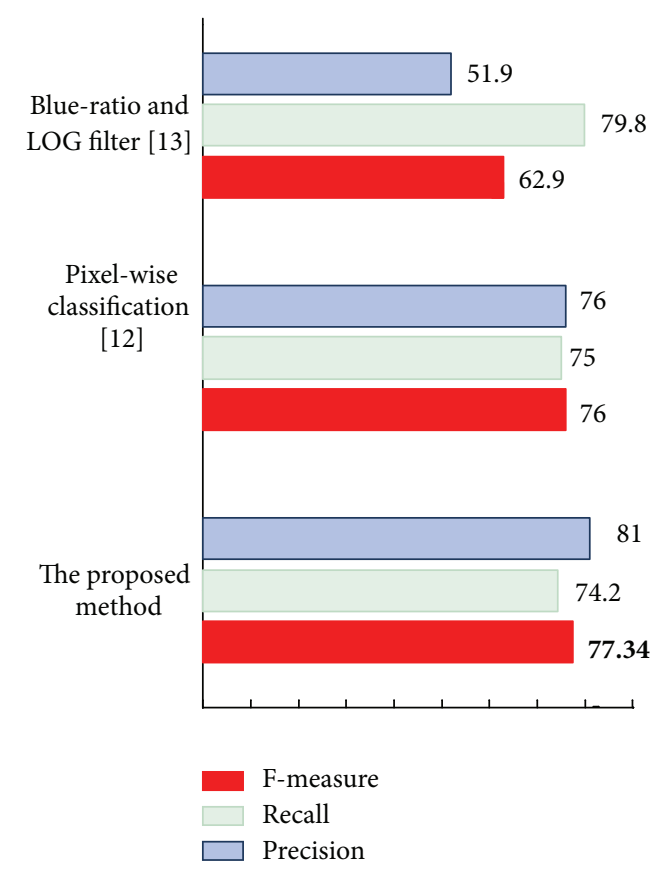

Figure 7: Result of mitosis detection in different algorithms.

compared with results of other mitosis detection systems in Figure 7 in which our system results in 77.34\% F-measure.

As seen in Figure 7, in the proposed system, because of the use of nonmitosis candidate reduction algorithm, the output FP is very low and this fact leads to the improvement of precision and F-measure.

The result of the proposed mitosis detection system for a breast cancer histopathology image is shown in Figure 4. 
In Figure 4(a), there are two mitoses, while the number of extracted candidates by ML algorithm is about 356 candidates (Figure $4(\mathrm{~b})$ ). Because of this fact that, in candidate extraction stage, the number of nonmitosis candidates is much more than mitosis candidates, classification results tend to nonmitosis or false positives. To overcome this problem, by employing the proposed candidate selection algorithm, the number of candidates is reduced to 97 (Figure 4(c)). According to results, about $72.75 \%$ of nonmitosis candidates are omitted. In fact about $72.75 \%$ of the candidates of sample histopathology image that can be false positive are reduced. In Figure 4(d) the result of mitosis detection by the proposed system is shown. The green circles show true positives and red circles show false positive results.

\section{Conclusion}

In this paper, an intelligent system for mitosis detection in breast cancer histopathology slide images is proposed. In the proposed system, by using ML algorithm that estimates the pdfs of mitosis and nonmitosis grey levels, the potential mitosis candidates are extracted. As the most number of extracted candidates are nonmitosis and to reduce the number of false positives, a new candidate selection algorithm is proposed. In this algorithm, by defining a cost function and minimizing it using TLBO algorithm, the large number of nonmitosis candidates is removed. Then by using different feature extraction methods, colour, shape, and texture features are extracted and by training a SVM classifier with different kernels, the mitosis is detected. Experimental results demonstrate that the proposed mitosis detection system has a performance of 77.34 $\%$ F-measure and outperforms related works in the literature.

\section{Conflict of Interests}

The authors declare that there is no conflict of interests regarding the publication of this paper.

\section{References}

[1] National Cancer Institute (NCI), http://www.cancer.gov/cancertopics.

[2] H. J. Bloom and W. W. Richardson, "Histological grading and prognosis in breast cancer; a study of 1409 cases of which 359 have been followed for 15 years," British Journal of Cancer, vol. 11, no. 3, pp. 359-377, 1957.

[3] S. Naik, S. Doyle, S. Agner, A. Madabhushi, M. Feldman, and J. Tomaszewski, "Automated gland and nuclei segmentation for grading of prostate and breast cancer histopathology," in Proceedings of the 5th IEEE International Symposium on Biomedical Imaging: From Nano to Macro (ISBI '08), pp. 284287, Paris, France, May 2008.

[4] J. R. Dalle, W. K. Leow, D. Racoceanu, A. E. Tutac, and T. C. Putti, "Automatic breast cancer grading of histopathological images," in Proceedings of the IEEE International Conference on Engineering in Medicine and Biology Society, pp. 3052-3055, 2008.

[5] A. E. Tutac, D. Racoceanu, T. Putti, W. Xiong, W. Leow, and V. Cretu, "Knowledge-guided semantic indexing of breast cancer histopathology images," in Proceedings of the IEEE International conference on In BioMedical Engineering and Informatics, vol. 2, pp. 107-112, May 2008.

[6] L. Latson, B. Sebek, and K. A. Powell, "Automated cell nuclear segmentation in color images of hematoxylin and eosin-stained breast biopsy," Analytical and Quantitative Cytology and Histology, vol. 25, no. 6, pp. 321-331, 2003.

[7] M. Veta, P. J. van Diest, R. Kornegoor, A. Huisman, M. A. Viergever, and J. P. W. Pluim, "Automatic nuclei segmentation in H\&E stained breast cancer histopathology images," PLoS ONE, vol. 8, no. 7, Article ID e70221, 2013.

[8] E. Cosatto, M. Miller, H. P. Graf, and J. S. Meyer, "Grading nuclear pleomorphism on histological micrographs," in Proceedings of the 19th International Conference on Pattern Recognition (ICPR '08), pp. 1-4, Tampa, Fla, USA, December 2008.

[9] A. Fakhrzadeh, E. Spörndly-Nees, L. Holm, and C. L. L. Hendriks, "Analyzing tubular tissue in histopathological thin sections," in Proceedings of the 14th International Conference on Digital Image Computing Techniques and Applications (DICTA '12), pp. 1-6, Fremantle, Australia, December 2012.

[10] A. Basavanhally, E. Yu, J. Xu et al., "Incorporating domain knowledge for tubule detection in breast histopathology using O'Callaghan neighborhoods," in Medical Imaging: ComputerAided Diagnosis, vol. 7963 of Proceedings of SPIE, Lake Buena Vista, FLa, USA, February 2011.

[11] A. M. Khan, H. El-Daly, and N. M. Rajpoot, "A gamma-gaussian mixture model for detection of mitotic cells in breast cancer histopathology images," in Proceedings of the IEEE International conference on Pattern Recognition, pp. 149-152, 2012.

[12] C. Sommer, L. Fiaschi, F. A. Hamprecht, and D. W. Gerlich, "Learning-based mitotic cell detection in histopathological images," in Proceedings of the 21st International Conference on Pattern Recognition (ICPR '12), pp. 2306-2309, November 2012.

[13] H. Irshad, S. Jalali, L. Roux, D. Racoceanu, L. J. Hwee, and G. Le Naour, "Automated mitosis detection using texture, SIFT features and HMAX biologically inspired approach," Journal of Pathology Informatics, vol. 4, p. 12, 2013.

[14] D. C. Cireşan, A. Giusti, L. M. Gambardella, and J. Schmidhuber, "Mitosis detection in breast cancer histology images with deep neural networks," in Medical Image Computing and Computer-Assisted Intervention, pp. 411-418, Springer, Berlin, Germany, 2013.

[15] C. H. Huang and H. K. Lee, "Automated mitosis detection based on exclusive independent component analysis," in Proceedings of the IEEE International Conference on Pattern Recognition, pp. 1856-1859, 2012.

[16] C. Huang and H. Lee, "Automated mitosis detection based on exclusive independent component analysis," in Proceedings of the IEEE 21st International Conference on Pattern Recognition (ICPR '12), pp. 1856-1859, November 2012.

[17] C. Lu and M. Mandal, "Towards automatic mitotic cells detection and segmentation in multi-spectral histopathological images," IEEE Journal of Biomedical and Health Informatics, vol. 18, no. 2, pp. 594-605, 2013.

[18] A. Tashk, M. S. Helfroush, H. Danyali, and M. AkbarzadehJahromi, "A novel CAD system for mitosis detection using histopathology slide images," Journal of Medical Signals and Systems, vol. 4, no. 2, pp. 139-149, 2014.

[19] A. Tashk, M. S. Helfroush, H. Danyali, and M. Akbarzadehjahromi, "A CAD mitosis detection system from breast cancer 
histology images based on fused features," in Proceedings of the 22nd Iranian Conference on Electrical Engineering (ICEE '14), Tehran, Iran, May 2014.

[20] A. Tashk, M. S. Helfroush, H. Danyali, and M. Akbarzadehjahromi, "An automatic mitosis detection method for breast cancer histopathology slide images based on objective and pixel-wise textural features classification," in Proceedings of the 5th Conference on Information and Knowledge Technology (IKT '13), pp. 406-410, Shiraz, Iran, May 2013.

[21] R. V. Rao, V. J. Savsani, and D. P. Vakharia, "Teaching-learningbased optimization: a novel method for constrained mechanical design optimization problems," Computer-Aided Design, vol. 43, no. 3, pp. 303-315, 2011.

[22] R. M. Haralick, K. Shanmugam, and I. H. Dinstein, "Textural features for image classification," IEEE Transactions on Systems, Man and Cybernetics, vol. 3, no. 6, pp. 610-621, 1973.

[23] B. V. Dasarathy and E. B. Holder, "Image characterizations based on joint gray level-run length distributions," Pattern Recognition Letters, vol. 12, no. 8, pp. 497-502, 1991.

[24] Z. Guo, L. Zhang, and D. Zhang, "A completed modeling of local binary pattern operator for texture classification," IEEE Transactions on Image Processing, vol. 19, no. 6, pp. 1657-1663, 2010.

[25] M. Torabi, S. Razavian, R. Vaziri, and B. Vosoughi-Vahdat, "A Wavelet-packet-based approach for breast cancer classification," in Proceedings of the 33rd Annual International Conference of the IEEE Engineering in Medicine and Biology Society (EMBS '11), pp. 5100-5103, Boston, Mass, USA, September 2011.

[26] C. Palm and T. M. Lehmann, "Classification of color textures by Gabor filtering," Machine Graphics and Vision, vol. 11, pp. 195220, 2002.

[27] B. E. Boser, I. M. Guyon, and V. N. Vapnik, "A training algorithm for optimal margin classifiers," Proceedings of the Annual WorkShop on Computational Learning Theory, pp. 144-152, 1992.

[28] Mitosis detection in breast cancer histological images. An ICPR 2012 contest, http://ipal.cnrs.fr/ICPR2012. 

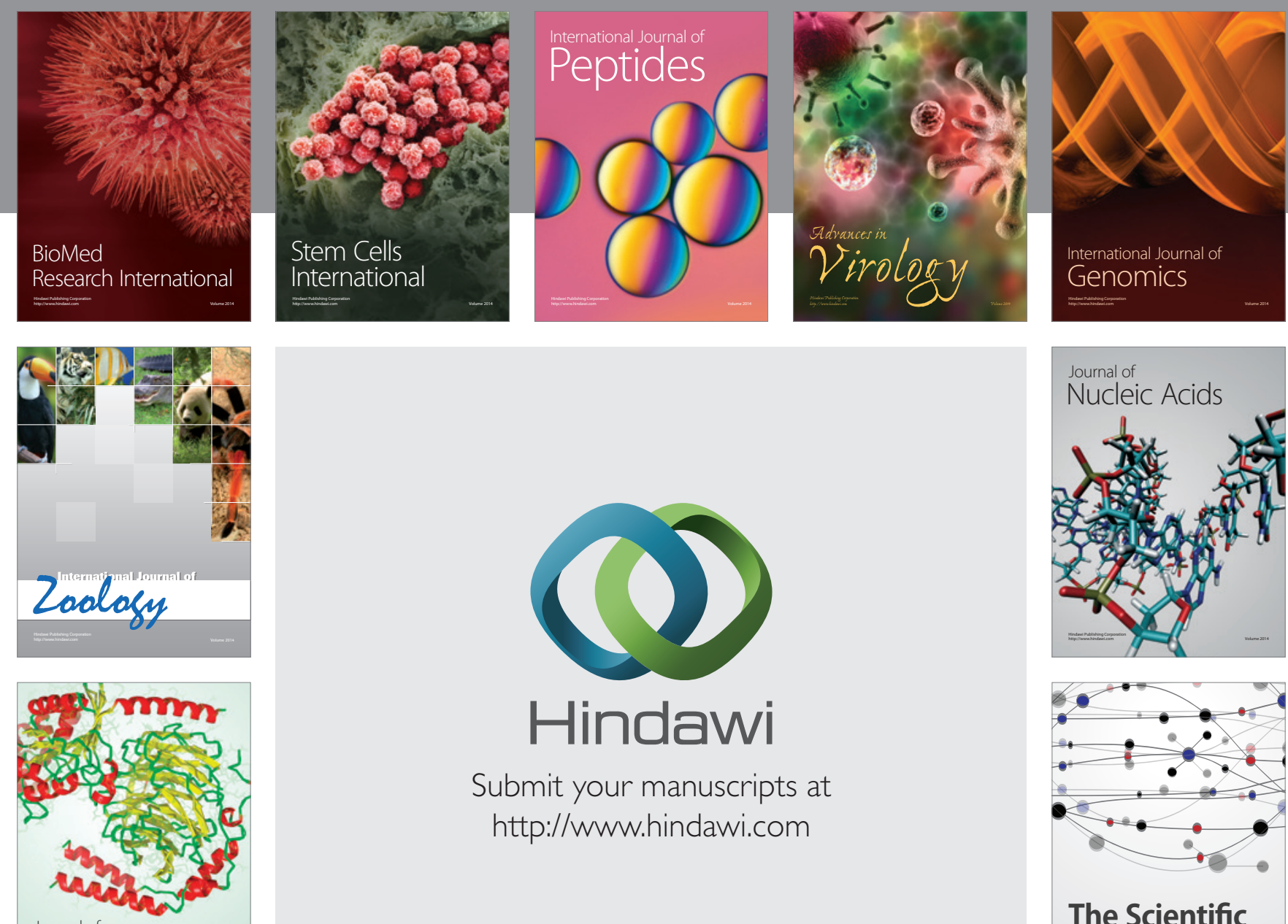

Submit your manuscripts at

http://www.hindawi.com

Journal of
Signal Transduction
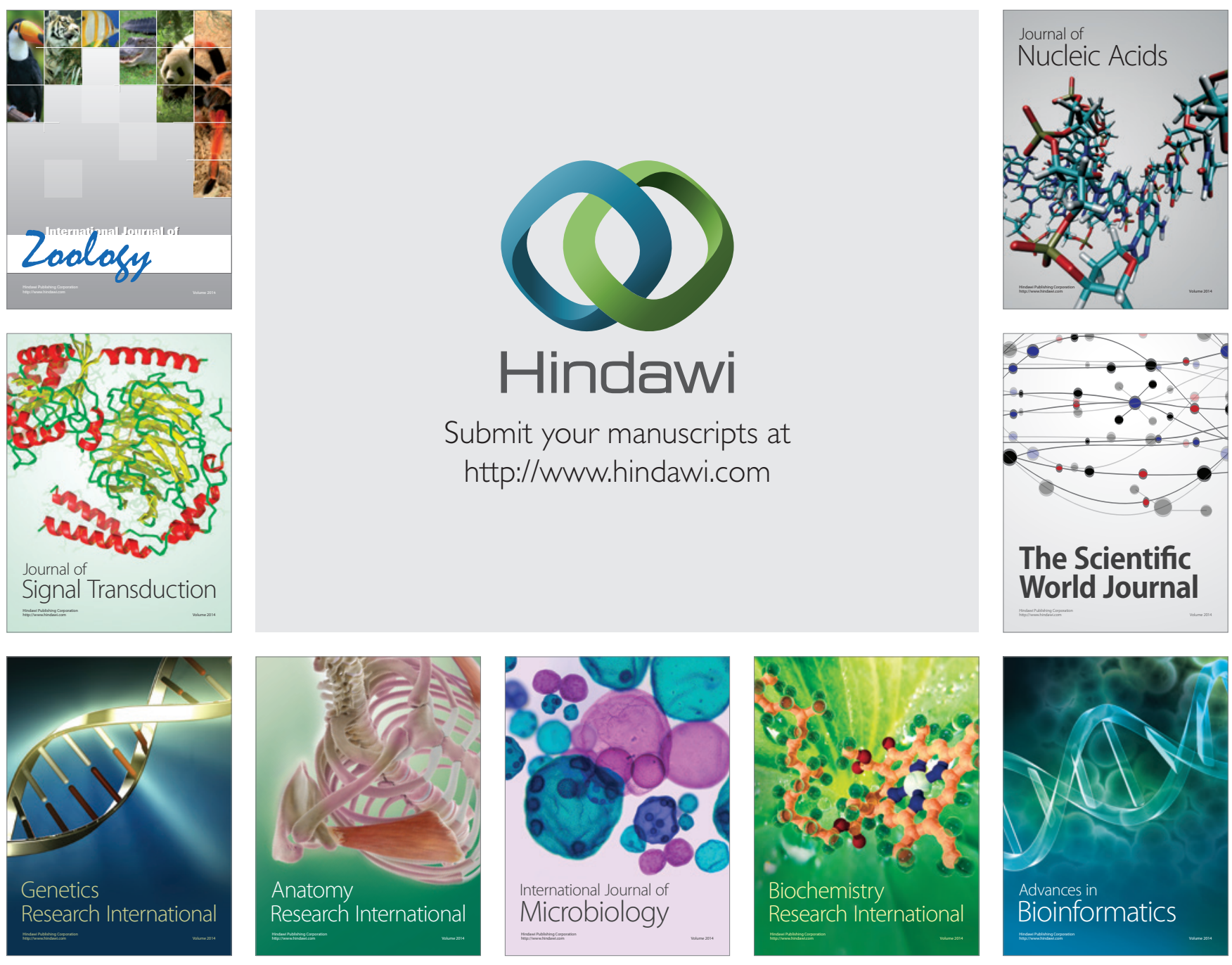

The Scientific World Journal
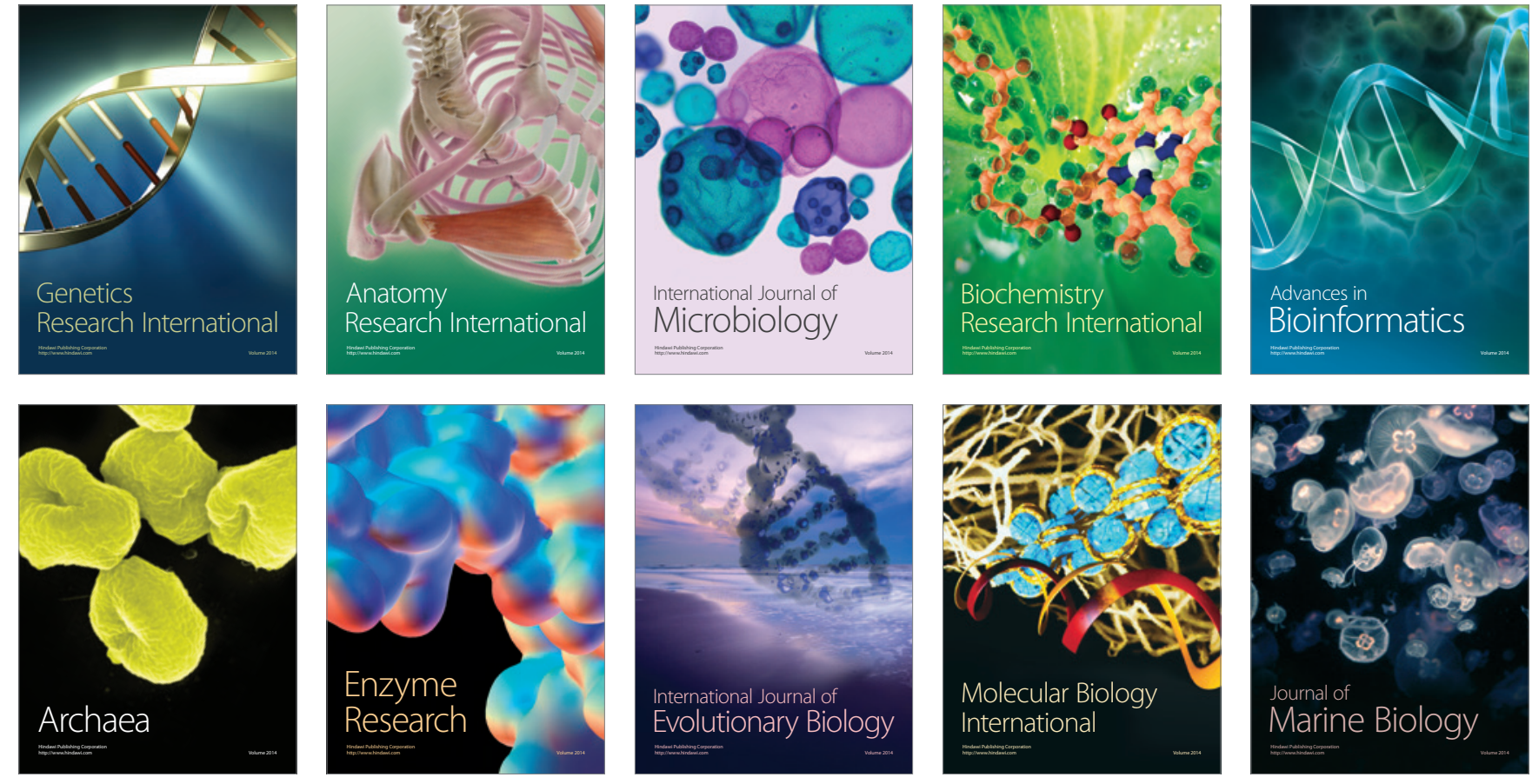